\title{
UNIQUENESS AND STABILITY OF NONNEGATIVE SOLUTIONS FOR SEMIPOSITONE PROBLEMS IN A BALL
}

\author{
ISMAEL ALI, ALFONSO CASTRO, AND R. SHIVAJI
}

(Communicated by Barbara L. Keyfitz)

\begin{abstract}
We study the uniqueness and stability of nonnegative solutions for classes of nonlinear elliptic Dirichlet problems on a ball, when the nonlinearity is monotone, negative at the origin, and either concave or convex.
\end{abstract}

\section{INTRODUCTION}

In this paper we consider the boundary value problem

$$
\begin{aligned}
& \Delta u+\lambda f(u)=0 \quad \text { in } \Omega, \\
& u=0 \text { on } \partial \Omega \text {, }
\end{aligned}
$$

where $\Delta$ is the Laplacian operator, $\Omega$ is the unit ball in $\mathbf{R}^{n}, n \geq 2$, and $\lambda>0$ is a parameter. We also assume that $f:[0, \infty) \rightarrow \mathbf{R}$ is monotonically increasing and that

$$
f(0)<0 \text { (semipositone) and } f(u)>0 \text { for some } u>0 .
$$

We will denote by $F$ the primitive of $f$ with $F(0)=0$.

Our main results are

Theorem A. If $f$ is concave then there exists $\mu_{1}$ such that if $\lambda>\mu_{1}$ then (1.1)(1.2) has at most one nonnegative solution. Furthermore, there exists $\mu_{2}$ such that all nonnegative solutions for $\lambda>\mu_{2}$ are stable.

Theorem B. If $f$ is convex, $\lim _{t \rightarrow \infty}(f(t) / t)=\infty$, there exists $k \in(0,1)$ such that

$$
\Lambda=\lim _{t \rightarrow \infty}(t / f(t))^{n / 2}\{2 n F(k t)-(n-2) t f(t)\}=\infty,
$$

and $\psi(t):=f(t) /\left(t f^{\prime}(t)-f(t)\right)$ is an increasing function, then there exists $\mu_{3}$ such that if $\lambda<\mu_{3}$ then (1.1)-(1.2) has at most one nonnegative solution.

The case $n=1$ was studied in [4] where, in particular, it was shown that Theorem $A$ is true for positive solutions but not for nonnegative solutions. Also in [4], Theorem B for $n=1$ was proven and an extensive study of existence

Received by the editors March 6, 1991 and, in revised form, July 1, 1991.

1991 Mathematics Subject Classification. Primary 34B15, 35 J65.

This research was partially supported by NSF grant DMS-8905936 and by the Texas Advanced Research Program. 
results was established. Existence results for $n>1$ can be found in [3, 5, 9-11]. For nonexistence results see [1] and for unstability of positive solutions in the convex case see [2]. Uniqueness and stability results have yet to be addressed, which we do here.

Our methods involve shooting arguments, estimates at and near the boundary, Pohozaev's identity, and comparison arguments. The assumption $f(0)<0$ makes it difficult to establish the existence of a minimal nonnegative solution, unlike in the case of positone problems $(f(0)>0)$.

Due to the results of [6] we know that nonnegative solutions to $(1.1)-(1.2)$ are positive in $\Omega$ and hence are radially symmetric (see [7]). Thus it is sufficient to study the equation

$$
\begin{gathered}
v^{\prime \prime}+((n-1) / r) v^{\prime}+\lambda f(v)=0, \\
v^{\prime}(0)=0, \\
v(1)=0 .
\end{gathered}
$$

For future reference we note that, because of $(1.3)$, there exist positive real numbers $\beta<\theta$ with

$$
f(\beta)=F(\theta)=0 .
$$

The number $\theta$ plays a crucial role in the study of positive solutions to (1.1)(1.2) as every positive solution to (1.4)-(1.6) satisfies

$$
v(0)>\theta \text {. }
$$

Indeed, if $v$ is a solution to $(1.4)-(1.6)$ nonnegative in $[0,1)$ then multiplying (1.4) by $r^{n} v^{\prime}$ and integrating on $[0,1]$ we have

$$
\left(v^{\prime}(1)\right)^{2}+\int_{0}^{1}(n-2) r^{n-1}\left(v^{\prime}(r)\right)^{2} d r=2 n \lambda \int_{0}^{1} r^{n-1} F(v(r)) d r .
$$

From (1.9) we clearly see that the integrand on the right-hand side must be positive for some $r$. Since $F$ is an increasing function on $[\beta, \infty)$ and $v$ is radially decreasing, we see that $(1.8)$ holds.

We will give preliminaries in $\S \S 2$ and 3 and prove our main theorems in $\S 4$.

\section{Preliminary lemmas, $f$ concave}

Throughout this section we assume that $f$ is concave.

Lemma 2.1. There exist positive constants $\lambda_{2}$ and $M_{2}$ such that if $v$ is a positive solution to (1.4)-(1.6) with $\lambda>\lambda_{2}$ then

$$
\left|v^{\prime}(1)\right|>M_{2} \lambda \text {. }
$$

Proof. From [1] it follows that there exists a positive real number $M$ independent of $\lambda$ such that if $v$ is a positive solution to (1.4)-(1.6) then $v\left(t_{0}\right)=\beta / 2$ for some $t_{0} \in(0,1)$ and

$$
1-t_{0} \leq M \lambda^{-1 / 2}
$$

From (2.2) and the mean value theorem we see that there exists $t^{*} \in\left[t_{0}, 1\right]$ with $\left|v^{\prime}\left(t^{*}\right)\right| \geq(\beta /(2 M)) \lambda^{1 / 2}$. Because the positive solutions to (1.4)-(1.6) are radially decreasing and $f$ is negative on $[0, \beta)$, we see that

$$
\left|v^{\prime}(s)\right| \geq(\beta /(2 M)) \lambda^{1 / 2} \text { for all } s \in\left[t_{1}:=v^{-1}(\beta), t^{*}\right] .
$$


Integrating $v^{\prime}$ on $\left[t_{1}, t_{0}\right]$ and using (2.2)-(2.3), we see that

$$
1-t_{1} \leq 2 M \lambda^{-1 / 2} \text {. }
$$

Let $\mu \in(\beta, \theta)$ be such that $\beta+\left(\beta^{2} /\left(64 M^{2} f(\mu)\right)\right) \geq \mu$. Let $t_{2} \in(0,1)$ be such that $v\left(t_{2}\right)=\mu$. The existence of such a $t_{2}$ follows from (1.8).

Now we show that $t_{2} \geq t_{1}-(\beta /(8 M f(\mu))) \lambda^{-1 / 2}$ for $\lambda$ large. Suppose not. Integrating (1.4) on $\left[t, t_{1}\right]$ for $t \in\left[t_{2}, t_{1}\right]$ we have (see (2.3))

$$
\begin{aligned}
t^{n-1} v^{\prime}(t) & =t_{1}^{n-1} v^{\prime}\left(t_{1}\right)+\lambda \int_{t}^{t_{1}} s^{n-1} f(v(s)) d s \\
& \leq-(\beta /(4 M)) \lambda^{1 / 2}+\lambda\left(t_{1}-t\right) f(\mu),
\end{aligned}
$$

where we used that $t_{1}^{n-1}$ is bounded below by $\frac{1}{2}$ as long as $\lambda$ is sufficiently large (see (2.4)). Thus if $t \geq t_{1}-(\beta /(8 M f(\mu))) \lambda^{-1 / 2}$ then $(\beta /(8 M)) \lambda^{1 / 2} \leq-v^{\prime}(t)$. By our choice of $\mu$ we have $v\left(t_{1}-(\beta /(8 M f(\mu))) \lambda^{-1 / 2}\right) \geq \mu$, which contradicts the definition of $t_{2}$. Thus there exists $\lambda_{3}$ such that for $\lambda \geq \lambda_{3}$ we have

$$
t_{1}-t_{2} \leq(\beta /(8 M f(\mu))) \lambda^{-1 / 2} .
$$

Because of (2.4) and (2.6), for $\lambda$ sufficiently large $t_{2}^{n}>\frac{1}{2}$. Thus for $t \in\left[t_{2}, 1\right]$ we obtain

$$
\begin{aligned}
t^{n-1} v^{\prime}(t) & =-\lambda \int_{0}^{t_{2}} s^{n-1} f(v(s)) d s-\lambda \int_{t_{2}}^{t} s^{n-1} f(v(s)) d s \\
& \leq-\lambda\left(f(\mu) t_{2}^{n}-\max \{|f(0)|, f(\mu)\}\left(1-t_{2}^{n}\right)\right) / n \\
& \leq-\lambda f(\mu) / 2 n+K \lambda^{1 / 2},
\end{aligned}
$$

where $K$ is independent of $\lambda$. From (2.7) we see that there exists $\lambda_{2} \geq \lambda_{3}$ and $M_{2}>0$ such that if $\lambda \geq \lambda_{2}$ then

$$
\left|v^{\prime}(t)\right| \geq M_{2} \lambda \text { for all } t \in\left[t_{2}, 1\right] .
$$

In particular,

$$
\left|1-t_{2}\right| \leq \mu\left(M_{2} \lambda\right)^{-1} .
$$

With inequality (2.8) the lemma is proven.

Corollary 2.2. Given $C>\mu$ there exists $\lambda(C)$ such that if $\lambda>\lambda(C)$ and $v$ is a positive solution to (1.4)-(1.6) then for some $T \in(0,1), v(T)=C$ and $1-T=O\left(\lambda^{-1}\right)$.

Proof. Because of (2.9), we can assume that $t_{2}>\frac{3}{4}$. Thus for all $t \in\left[\frac{1}{4}, t_{2}\right]$ we have

$$
-t^{n-1} v^{\prime}(t)=\lambda \int_{0}^{t} s^{n-1} f(v(s)) d s \geq \lambda f(\mu) t^{n} / n \geq \gamma \lambda,
$$

where $\gamma=f(\mu) /\left(4^{n} n\right)$. Now for all $t \in\left[\frac{1}{4}, \frac{3}{4}\right]$ we obtain

$$
v(t)=\mu-\int_{t}^{t_{2}} v^{\prime}(s) d s \geq \mu+\gamma \lambda\left(t_{2}-t\right) \geq \mu+\gamma \lambda\left(t_{2}-\frac{3}{4}\right) .
$$

Hence there exists $T$ such that $v(T)=c$ for $\lambda$ large and $1-T=O\left(\lambda^{-1}\right)$. 
Lemma 2.3. If (1.4)-(1.6) has a positive solution for all $\lambda$ large then $\lim _{t \rightarrow \infty} f^{\prime}(t)$ $=0$.

Proof. Suppose $\lim _{t \rightarrow \infty} f^{\prime}(t)=A>0$. Let $t_{1}$ be as in Lemma 2.1. Without loss of generality, we can assume that $t_{1}>\frac{1}{2}$ (see (2.4)). Let $m(r)$ denote an eigenfunction corresponding to the smallest eigenvalue $\sigma\left(t_{1}\right)$ of $-\Delta$ with Dirichlet boundary condition on the ball of radius $t_{1}$. Thus

$$
\int_{0}^{t_{1}} s^{n-1}(\lambda(g(w) / w)-\sigma) w m d s=0
$$

where $g(t)=f(t+\beta)$ and $w(t)=v(t)-\beta$. Since $f^{\prime \prime} \leq 0$, the function $g(w) / w$ is nonincreasing. Thus if $\lambda>\left(\sigma\left(\frac{1}{2}\right) / A\right)$ we have a contradiction, which proves the lemma.

\section{Preliminaries, $f$ convex}

Throughout this section we assume $f$ to satisfy the assumptions of Theorem B.

Let $z(r, \lambda, d)$ denote the solution to the initial value problem

$$
\begin{gathered}
z^{\prime \prime}+((n-1) / r) z^{\prime}+\lambda f(z)=0, \\
z(0)=d, \\
z^{\prime}(0)=0 .
\end{gathered}
$$

Suppose, in addition, that $z$ is positive in $[0,1)$, and that $z(1)=0$. Thus $z$ is a positive solution to (1.4)-(1.6). Because $\psi$ is an increasing function (see Theorem B), for each $\gamma \in[0,2 \psi(z(0))$ the function $\sigma(r):=\phi(z(r), \gamma):=$ $-(2+\gamma) f(z(r))+\gamma z(r) f^{\prime}(z(r))$ has exactly one zero $r^{*}(\gamma)$. This follows from the facts that $\sigma(1)>0, \sigma(0)=-(2+\gamma) f(d)+\gamma d f^{\prime}(d)=-2 f(d)+$ $\gamma\left\{d f^{\prime}(d)-f(d)\right\}<0$, and that $\gamma=2 \psi\left(z\left(r^{*}\right)\right)$ with $\psi$ increasing and $z$ decreasing. Moreover, $r^{*}$ is a continuous function of $\gamma$ and

$$
\phi(z(r), \gamma)<0 \text { for } r<r^{*}(\gamma) \text { and } \phi(z(r), \gamma)>0 \text { for } r>r^{*}(\gamma) .
$$

Let $h(r)=-r z^{\prime}(r) / z(r)$. From Pohozaev's identity we have

$$
h^{\prime}(r)=\lambda\left\{2 r^{1-n} J+r(z f(z)-2 F(z))\right\} / z^{2},
$$

where $J=\int_{0}^{r} s^{n-1}(n F(z)-((n-2) / 2) z f(z)) d s$. Since $f$ is convex and $f(0)<$ 0 , we have $z f(z)-2 F(z) \geq 0$. Also (see [5, (3.3)]) because $\Lambda=\infty$ we see that $J \geq 0$. Hence $h^{\prime} \geq 0$. Now letting $H(r):=2 \psi(z(r))-h(r)$ we see that $H$ is strictly decreasing, $H(0)>0$, and $\lim _{r \rightarrow 1} H(r)=-\infty$. Let $r_{0} \in(0,1)$ be such that $H\left(r_{0}\right)=0$. Thus if $\gamma_{0}=h\left(r_{0}\right)$ then $\gamma_{0}=2 \psi\left(z\left(r_{0}\right)\right)$, i.e., $r_{0}=r^{*}\left(\gamma_{0}\right)$. Hence

$$
\begin{gathered}
\phi\left(z(r), \gamma_{0}\right)<0 \text { and } y(r):=r z^{\prime}(r)+\gamma_{0} z(r)>0 \text { for } r<r_{0}, \\
\phi\left(z(r), \gamma_{0}\right)>0 \text { and } y(r)<0 \text { for } r>r_{0} .
\end{gathered}
$$

An elementary calculation shows that

$$
\begin{aligned}
y^{\prime \prime}+((n-1) / r) y^{\prime}+\lambda f^{\prime}(z) y & =\lambda \phi\left(z(r), \gamma_{0}\right), \\
y^{\prime}(0) & =0 .
\end{aligned}
$$


Let $w$ denote the solution to

$$
\begin{gathered}
w^{\prime \prime}+((n-1) / r) w^{\prime}+\lambda f^{\prime}(z) w=0, \\
w(0)=1, \quad w^{\prime}(0)=0 ;
\end{gathered}
$$

that is, $w$ is the derivative with respect to $d$ of $z(r, \lambda, d)$.

Now we are ready to prove.

Lemma 3.1. Under the above assumptions $w$ has exactly one zero in $[0,1]$ and $w(1)<0$.

Proof. First we show that $w$ is positive on $\left[0, r^{*}\left(\gamma_{0}\right)=r_{0}\right]$. In fact, suppose $w$ has a zero in the latter interval. Let $c$ be the smallest positive zero of $w$. Multiplying (3.7) by $r^{n-1} w$ and (3.9) by $r^{n-1} y$ and integrating on $[0, c]$ we have

$$
c^{n-1} w^{\prime}(c) y(c)=-\lambda \int_{0}^{c} \phi\left(z(r), \gamma_{0}\right) r^{n-1} w(r) d r .
$$

Since (3.11) contradicts (3.6) we see that $w$ is positive on $\left[0, r_{0}\right]$. On the other hand, if $w$ has its first two zeros $p<q$ in the interval $\left(r_{0}, 1\right]$, then arguing as in (3.11) we have

$$
q^{n-1} w^{\prime}(q) y(q)-p^{n-1} w^{\prime}(p) y(p)=-\lambda \int_{p}^{q} \phi\left(z(r), \gamma_{0}\right) w(r) r^{n-1} d r
$$

which contradicts (3.6) and the fact that $p$ and $q$ are the first two zeros of $w$. Thus $w$ has at most one zero on $\left(r_{0}, 1\right]$. Let us see that such a zero exists in $\left(r_{0}, 1\right)$. Since $z$ satisfies

$$
\begin{gathered}
z^{\prime \prime}+((n-1) / r) z^{\prime}+\lambda f^{\prime}(z) z=\lambda\left(z f^{\prime}(z)-f(z)\right), \\
z>0 \text { on }[0,1), \quad z(1)=0=z^{\prime}(0),
\end{gathered}
$$

and since $f$ is convex, the right-hand side of (3.13) is positive. But multiplying (3.13) by $r^{n-1} w$ and (3.9) by $r^{n-1} z$ and integrating on $[0,1]$ we obtain

$$
z^{\prime}(1) w(1)=\lambda \int_{0}^{1}\left(z f^{\prime}(z)-f(z)\right) r^{n-1} w d r
$$

and know that $z^{\prime}(1) \leq 0$. Hence from (3.15) $w$ must have a zero in $\left(r_{0}, 1\right)$ and $w(1)<0$. Thus Lemma 3.1 is proven.

Finally, an elementary calculation shows that

$$
z(r / \alpha, \lambda, d)=z\left(r, \lambda / \alpha^{2}, d\right) \text { for all } \alpha>0 .
$$

Differentiating the latter identity with respect to $\alpha$ and replacing $\alpha$ by 1 we have

$$
r z^{\prime}(r, \lambda, d)=2 \lambda z_{\lambda}(r, \lambda, d)
$$

\section{Proof of Theorems A AND B}

Proof of Theorem A. Suppose (1.1)-(1.2) has positive solutions for arbitrarily large values of $\lambda$. From Lemma 2.3 we see that there exist positive constants $a \geq \mu$ and $b$ such that

$$
f(t) \geq t f^{\prime}(t)+b \quad \text { for all } t \geq a .
$$


By Corollary 2.2 we see that there exists $t_{3} \leq t_{2}$ such that $v\left(t_{3}\right)=a$ and

$$
\left|t_{3}-1\right| \leq O\left(\lambda^{-1}\right)
$$

for any $v$ positive solution to $(1.4)-(1.6)$. Let us see now that if $y:[0,1] \rightarrow \mathbf{R}$ satisfies

$$
\begin{gathered}
y^{\prime \prime}+\left((n-1) y^{\prime} / r\right)+\lambda f^{\prime}(v) y=0, \\
y(0)=1, \\
y^{\prime}(0)=0,
\end{gathered}
$$

then $y>0$ on $[0,1]$. Suppose not. Let $T \in(0,1]$ be the first zero of $y$. Multiplying (4.3) by $r^{n-1} v$ and (1.4) by $r^{n-1} y$ and integrating on $[0, T]$ we have

$$
0=T^{n-1} y^{\prime}(T) v(T)-\lambda \int_{0}^{T} r^{n-1}\left(f(v)-f^{\prime}(v) v\right) y d r
$$

Since $y^{\prime}(T) \leq 0$ and $f(v)>f^{\prime}(v) v$ on $\left[0, t_{3}\right]$ we see that $T>t_{3}$. Thus from (4.1), (4.6), and the fact that $y$ is nonincreasing on $(0, T]$, we have

$$
\begin{aligned}
\left(t_{3}^{n} / n\right) b y\left(t_{3}\right) & \leq \int_{0}^{t_{3}} r^{n-1}\left(f(v)-v f^{\prime}(v)\right) y d r \\
& \leq \int_{t_{3}}^{T} r^{n-1}\left(f^{\prime}(v) v-f(v)\right) y d r \\
& \leq y\left(t_{3}\right) O\left(\lambda^{-1}\right) .
\end{aligned}
$$

But from (4.2) we see that the latter inequality cannot hold for $\lambda$ large. Therefore $y$ is positive on $[0,1]$.

Suppose now that for $\lambda$ large $(1.4)-(1.6)$ has two solutions positive on $[0,1)$. Let them be $v$ and $w$. By the uniqueness of solutions for initial value problems we see that $v(0) \neq w(0)$, say $v(0)>w(0)$. Taking $T=\min \{t \in[0,1]$; $w(t)=v(t)\}$ we see that $z=v-w$ is positive on $[0, T)$ and

$$
\begin{gathered}
z^{\prime \prime}+\left((n-1) z^{\prime} / r\right)+\lambda((f(v)-f(w)) /(v-w)) z=0, \\
z^{\prime}(0)=z(T)=0 .
\end{gathered}
$$

Since $f$ is concave $(f(v)-f(w)) /(v-w) \leq f^{\prime}(w)$ on $[0, T]$. This and the Sturm comparison theorem imply that $y$ must have a zero on $[0, T)$, which we have proven not to happen. Thus (1.4)-(1.6) has at most one positive solution.

Next suppose $u$ is a nonnegative solution for $\lambda$ large. Then consider the eigenvalue problem

$$
\begin{aligned}
\phi^{\prime \prime}+((n-1) / r) \phi^{\prime}+\left(\lambda f^{\prime}(u)+\lambda_{1}\right) \phi & =0, \\
\phi(0)=1, \quad \phi^{\prime}(0) & =0, \quad \phi(1)=0,
\end{aligned}
$$

where $\lambda_{1}$ is the smallest eigenvalue. If $\lambda_{1} \leq 0$ then by the Sturm comparison theorem $y$ must vanish in $[0,1]$ which is a contradiction. Hence $\lambda_{1}>0$ and thus $u$ is stable (see [8]), which proves Theorem $\mathrm{A}$. 
Proof of Theorem B. Let $z(r, \lambda, d)$ be as in $\S 3$. From Lemma 3.2 of [5], it follows that there exist $\lambda^{*}$ and $d^{*}$ such that

$$
\begin{aligned}
& \text { If } \lambda \in\left(0, \lambda^{*}\right) \text { and } d>d^{*} \text { then }(z(r, \lambda, d))^{2}+\left(z^{\prime}(r, \lambda, d)\right)^{2}> \\
& 0 \text { for all } r \in[0,1] \text {. }
\end{aligned}
$$

Let now $z\left(, \lambda_{0}, d_{0}\right)$ be a solution to $(1.4)-(1.6)$ positive in $[0,1)$ with $\lambda_{0}<$ $\lambda^{*}$. By further restricting $\lambda^{*}$ we can assume that if $\lambda_{0}<\lambda^{*}$ then $d_{0}>d^{*}$ (see [5, Lemma 3.2]). Hence from (3.17), (4.11), Lemma 3.1, and the implicit function theorem there exist $\varepsilon>0$ and a decreasing function $\Lambda:\left[d_{0}-\varepsilon, \infty\right) \rightarrow$ $\mathbf{R}$ such that $\Lambda\left(d_{0}\right)=\lambda_{0}$, and $z(, \Lambda(d), d)$ is a positive solution to (1.4)(1.6). Suppose now that for some $\lambda^{\prime} \in\left(0, \lambda^{*}\right)$ the problem (1.4)-(1.6) has two solutions $z\left(, \lambda^{\prime}, d_{1}\right)$ and $z\left(, \lambda^{\prime}, d_{2}\right)$. Without loss of generality we can assume that $d_{1}<d_{2}$. Let $\Lambda_{1}$ and $\Lambda_{2}$ be the functions whose graphs determine positive solutions to (1.4)-(1.6) and such that

$$
\Lambda_{1}\left(d_{1}\right)=\Lambda_{2}\left(d_{2}\right)=\lambda^{\prime}
$$

Since both $\Lambda_{1}$ and $\Lambda_{2}$ are decreasing functions, their graphs are disjoint. Let $\lambda^{\prime \prime}=\Lambda_{1}\left(d_{2}\right)$. Hence $\lambda^{\prime \prime}<\lambda^{\prime}$. Since by (3.17) and (4.11) the first zero of $z(, \lambda, d)$ is a decreasing function of $\lambda$, we see that $z\left(, \lambda^{\prime \prime}, d_{2}\right)$ has a zero in $(0,1)$, which contradicts that it is a positive solution to $(1.4)-(1.6)$, and Theorem $\mathrm{B}$ is proven.

\section{REMARKS}

Theorem B holds under less restrictive assumptions. Indeed, the reader can verify that the convexity condition may be replaced by suitable superlinear conditions. However, there remain a great deal of open questions. For example, in Theorem $B$ uniqueness of the positive solutions for arbitrary values of $\lambda$ remains unsettled even in the radial case. Also Theorems A and B need to be proven for general regions.

\section{REFERENCES}

1. K. J. Brown, A. Castro, and R. Shivaji, Nonexistence of radially symmetric nonnegative solutions for a class of semipositone problems, Differential Integral Equations 2 (1989), 541-545.

2. K. J. Brown and R. Shivaji, Instability of nonnegative solutions for a class of semipositone problems, Proc. Amer. Math. Soc. 112 (1991), 121-124.

3. A. Castro, J. B. Garner, and R. Shivaji, Existence results for a class of sublinear semipositone problems, Result. Math. (to appear).

4. A. Castro and R. Shivaji, Nonnegative solutions for a class of nonpositone problems, Proc. Roy. Soc. Edingburgh Sect. A 108 (1988), 291-302.

5. __ Nonnegative solutions for a class of radially symmetric nonpositone problems, Proc. Amer. Math. Soc. 106 (1989), 735-740.

6. __ Nonnegative solutions to a semilinear Dirichlet problem in a ball are positive and radially symmetric, Comm. Partial Differential Equations 14 (1989), 1091-1100.

7. B. Gidas, W. Ni, and L. Nirenberg, Symmetry and related properties via the maximum principles, Comm. Math. Phys. 68 (1979), 209-243.

8. D. H. Sattinger, Monotone methods in nonlinear elliptic and parabolic boundary value problems, Indiana Univ. Math. J. 21 (1972), 979-1000.

9. J. Smoller and A. Wasserman, Existence of positive solutions for semilinear elliptic equations in general domains, Arch. Rational Mech. Anal. 98 (1987), 229-249. 
10. G. Sweers, Semilinear elliptic eigenvalue problems, Doctoral Thesis, Univ. of Leiden, Netherlands, 1988.

11. S. Unsurangsie, Existence of a solution for a wave equation and an elliptic Dirichlet problem, Doctoral Thesis, Univ. of North Texas, 1988.

(I. Ali and A. Castro) Department of Mathematics, University of North Texas, Denton, TEXAs 76203-5116

E-mail address, A. Castro: acastro@untvax.bitnet

(R. Shivaji) Department of Mathematics, Mississippi State University, Mississippi State, MississiPPI 39762

E-mail address: rs1@ra.msstate.edu 